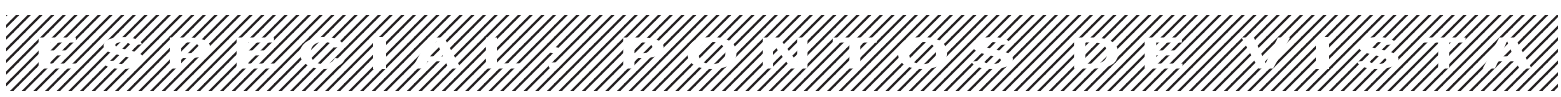

NOTA: as quatro comunicações a seguir foram escritas a convite do Editor deste número temático, em resposta à questão: “em sua opinião, em que direção - ou direções - ocorrerá o desenvolvimento da Psicologia Ambiental nos próximos anos?”.

\title{
Los desarrollos de la Psicología Ambiental en los próximos años
}

\author{
Juan Ignacio Aragonés \\ Universidad Complutense de Madrid, Espanha
}

$\mathrm{P}$ ara hablar del devenir de la Psicología Ambiental a medio plazo es necesario mirar hacia atrás para ver lo que ha sucedido en la corta historia de esta disciplina. En los años 1970 aparecieron numerosos trabajos a propósito de lo que se entendía en aquellos momentos por psicología ambiental, pero sin duda uno de los trabajos de mayor interés, a mi juicio, es el de Proshansky (1976). Este reconocido psicólogo social apostaba en aquel artículo por una psicología ambiental renunciando a la psicología social dado su carácter irrelevante como disciplina. Aquel importante texto sólo se podía escribir si se piensa en el gran desarrollo que tuvo la psicología social en los años 1950 y 1960. Las numerosas investigaciones de carácter básico que tuvieron lugar en esos momentos llevaron al desarrollo de metodologías nuevas que permitieron pensar en una psicología ambiental orientada por el problema. Es decir, se invirtió el conocimiento acumulado por la investigación psicosocial básica sobre cómo los problemas de la vida cotidiana afectaban a las personas en su relación con el medio ambiente.

Si se recurre a repasar otros tipos de documentos tales como los manuales de la psicología ambiental de los años 1970, las publicaciones de EDRA, o los números de la revista Environment and Behavior aparece una psicología ambiental fructífera enfocada hacia el estudio de la relación entre el hombre y el medio construido. Los desarrollos de esos años y posteriores se ven claramente reflejados en la magnífica obra de Stokols y Altman (1987). En ella se observan dos grandes dimensiones que estructuran sendos volúmenes. En la primera parte los autores estadounidenses recogen el acervo fundamental de conocimientos de la disciplina. Podría decirse que en estos capítulos se presentan los temas fundamentales que componían la disciplina, junto con sus desarrollos más consistentes. La segunda parte del texto permitió a autores con cierto prestigio internacional, ajenos al mundo estadounidense, presentar los trabajos que consideraban más interesantes en sus respectivos países. Es decir, la estructura de la obra mostró la hegemonía que los investigadores estadounidenses ejercían sobre la disciplina y que era seguida por los psicólogos ambientales del resto del mundo.

En trabajos más recientes, Stokols $(1995,1997)$ señala el carácter internacional de los desarrollos de la psicología ambiental, tratando de mostrar que a cada nicho ecológico o área geográfica le corresponde un tema de desarrollo. No obstante, análisis llevados a cabo por Aragonés (2000), en los que presta atención a la relación entre los "descriptores mayores” que establece la APA (American Psychological Association) en las publicaciones Environment and Behavior y Journal of Environmental Psychology y la procedencia del primer autor de cada artículo, no permiten aceptar el planteamiento de Stokols desde una perspectiva general ya que los resultados apuntan a que en EE.UU. se desarrolla prácticamente toda la psicología ambiental y sólo pequeños temas como los vinculados a algunos procesos básicos relacionados con la memoria y el aprendizaje o con la seguridad, son propios de la psicología ambiental europea. Puede decirse, por tanto, que la psicología ambiental que se haga en el futuro será la que se desarrolle en EE.UU.

Pero tras esta premisa convendría conocer qué dicen los estadounidenses sobre cuál será el futuro de la disciplina. Para ello puede echarse mano de los artículos de Stokols $(1995,1997)$ que se acaban de mencionar. En ellos el autor señala como temas de futuro las dimensiones psicológicas de la contaminación ambiental y del cambio global, los brotes de violencia en los niveles regionales e internacionales, el impacto de los cambios tecnológicos en el mundo del trabajo y en la vida familiar, las estrategias fundamentadas en el ambiente para la promoción de la salud comunitaria, y las implicaciones del aumento de la edad media de la población en el diseño ambiental y en la planificación comunitaria. Todos ellos se sitúan en la línea clásica de la psicología ambiental en cuanto los temas se orientan por el problema, pero no parece que estos sean los temas dominantes a tenor del debate que surge en el seno de la División 34 de la APA.

La discusión en Population and Environmental Psychology Bulletin deja traslucir la importancia de la preocupación ambiental como tema sobre el que pivotan los intereses actuales de la psicología ambiental. Este ámbito de estudio se extiende a otras latitudes europeas y latinoamericanas. Es decir, parece que se está ante un abandono mayoritario de los temas clásicos de la disciplina preocupados por la interacción con el medio construido y se está prestando atención al primer tema de futuro señalado por Stoklos.

Este breve análisis de la psicología ambiental de los tiempos pasados proyecta un futuro próximo en el que la 
carencia de investigación básica impedirá dar respuestas más precisas a las relaciones entre el ser humano y el medio ambiente construido y natural y, por tanto, se asistirá como mínimo al debilitamiento de la psicología ambiental más genuina. Una de las múltiples causas que llevará a esta posible situación es el intento, casi siempre frustrado, de aplicación con el que se trabaja en esta disciplina, que lleva a vaciarla de contenidos conceptuales y metodológicos propios.

A principios de los años 1970 era fácil investigar sobre los problemas de la relación entre el ser humano y el medio construido porque la psicología social, fundamentalmente, proporcionaba los conceptos y los procedimientos de trabajo, pero cuando esto dejó de suceder, se produjo una crisis en la psicología ambiental tradicional de la cual no se ha recuperado completamente. Esta barrera resulta difícil de salvar si además hay falta de recursos y escasa implantación institucional tal y como reconoce Sommer (1997) cuando repasa su biografía, siguiendo el hilo conductor del desarrollo de la psicología ambiental.

El impulso de los temas relacionados con la preocupación ambiental hunde sus raíces en la psicología social más clásica y, por tanto, abandona al ambiente como estímulo holístico de carácter sociofísico. Queda pues la psicología ambiental al albur de las modas: hoy es el ambientalismo, mañana otra problemática demandada, por supuesto, por la sociedad occidental.

\section{Referencias}

Aragonés, J. I. (2000). Proyecto docente. Documento no-publicado, Universidad Complutense, Madrid, España.

Proshansky, H. M. (1976). Environmental Psychology and real world. American Psychologist, 4, 303-310. [Traducido al español: Cuadernos de Psicología, 6, 172-186.]

Sommer, R. (1997). Benchmarks in Environmental Psychology. Journal of Environmental Psychology, 17, 1-10.

Stokols, D. (1995). The paradox of Environmental Psychology. American Psychologist, 50, 821-837.

Stokols, D. (1997). Directions of Environmental Psychology in the Twenty-First Century. In S. Wapner, J. Demick, T. Yamamoto \& T. Takahashi (Orgs.), Handbook of Japan-United States Environment-Behavior research. Toward a transactional approach (pp. 333-353). Nueva York: Plenum.

Stokols, D., \& Altman, I. (Orgs.). (1987), Handbook of Environmental Psychology (2 vols.). Nueva York: John Wiley \& Sons.

Juan Ignacio Aragonés, doutor em Psicologia pela Universidad Complutense de Madrid, Espanha, é professor titular nessa mesma universidade. Endereço para correspondência: Facultad de Psicologia, Campus de Somosaguas, Universidad Complutense, 28223 - Madrid, Espanha. Tel.: +34 91 394-2910. Fax: +34 91 3943189.E-mail: jiaragones@psi.ucm.es 